\title{
OSCILLATION OF SECOND ORDER DELAY DIFFERENTIAL EQUATIONS WITH NONLINEAR NONPOSITIVE NEUTRAL TERM
}

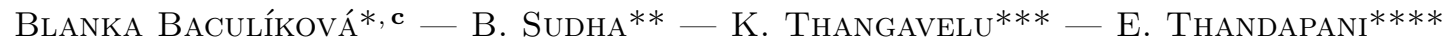 \\ (Communicated by Michal Fečkan)
}

\begin{abstract}
This paper deals with oscillation of a second order delay differential equations with a nonlinear nonpositive neutral term. Some new oscillation criteria and three examples are presented which improve and generalize the results reported in the literature.
\end{abstract}

$$
\text { (c) } 2022
$$

Mathematical Institute Slovak Academy of Sciences

\section{Introduction}

In this paper, we study the oscillatory behavior of a second order nonlinear neutral delay differential equations of the form

$$
\left(a(t)\left(x(t)-p(t) x^{\alpha}(\tau(t))\right)^{\prime}\right)^{\prime}+q(t) x^{\beta}(\sigma(t))=0, \quad t \geq t_{0}>0
$$

subject to the following conditions:

$\left(\mathrm{C}_{1}\right) 0<\alpha \leq 1$, and $\beta$ are ratio of odd positive integers;

$\left(\mathrm{C}_{2}\right) a \in C^{1}\left(\left[t_{0}, \infty\right),(0, \infty)\right)$, and $p, q \in C\left(\left[t_{0}, \infty\right),(0, \infty)\right)$ and $p(t)$ is such that $0<p(t) \leq p<1$ for all $t \geq t_{0}$;

$\left(\mathrm{C}_{3}\right) \tau \in C^{1}\left(\left[t_{0}, \infty\right), \mathbb{R}\right), \sigma \in C^{1}\left(\left[t_{0}, \infty\right), \mathbb{R}\right), \tau(t) \leq t, \sigma(t) \leq t, \tau^{\prime}(t)>0, \sigma^{\prime}(t)>0$, and $\lim _{t \rightarrow \infty} \tau(t)=\lim _{t \rightarrow \infty} \sigma(t)=\infty$.

By a solution of equation (1.1), we mean a function $x \in C\left(\left[T_{x}, \infty\right), \mathbb{R}\right), T_{x} \geq t_{0}$, which has the property $a(t)\left(x(t)-p(t) x^{\alpha}(\tau(t))\right)^{\prime} \in C^{1}\left(\left[T_{x}, \infty\right), \mathbb{R}\right)$ and satisfies equation 1.1 on $\left[T_{x}, \infty\right)$. We consider only those solutions $x$ of equation (1.1) which satisfy $\sup \{|x(t)|: t \geq T\}>0$ for all $T \geq T_{x}$, and assume that equation (1.1) possesses such solutions. As usual, a solution of equation (1.1) is called oscillatory if it has arbitrarily large zeros on $\left[T_{x}, \infty\right)$; otherwise it is called nonoscillatory.

In the last few years, there has been great interest in investigating the oscillatory behavior of solutions of different types of neutral differential equations since such equations have numerous applications in science and engineering, see $[8,9]$. We choose to investigate the oscillatory behavior of solutions of equation (1.1) since similar properties for delay differential equations with linear neutral term are studied in many papers, see $1,3,3,6,10,13,16,17]$ and the references cited therein.

2020 Mathematics Subject Classification: Primary 34C10, 34 K11.

Keywords: Oscillation, neutral differential equation, nonpositive sublinear neutral term.

${ }^{\mathbf{c}}$ Corresponding author. 
Very recently, in 2, 14, the authors considered equation (1.1) with $p(t)<0$ for all $t \geq t_{0}$, and established criteria for the oscillation of all solutions under the following condition

$$
\int_{t_{0}}^{\infty} \frac{\mathrm{d} t}{a(t)}=\infty .
$$

Motivated by the above observation, in this paper we derive some new oscillation results for the equation (1.1), which improve and complement those reported in $2,11,13,15$.

\section{Oscillation results}

In this section, we present sufficient conditions for the oscillation of all solutions of equation (1.1). Define

$$
\begin{aligned}
z(t) & =x(t)-p(t) x^{\alpha}(\tau(t)), \\
R(t) & =\int_{t_{1}}^{t} \frac{\mathrm{d} s}{a(s)} \quad \text { for } t \geq t_{1} \geq t_{0} .
\end{aligned}
$$

Note that from the assumptions and the form of equation [1.1p, it is enough to state and prove the results for the case of positive solutions since the proof of the other case is similar.

Lemma 2.1. Assume condition (1.2) holds. If $x$ is a positive solution of equation (1.1) then the corresponding function $z$ satisfies one of the following two cases:

(I) $z(t)>0, z^{\prime}(t)>0,\left(a(t) z^{\prime}(t)\right)^{\prime}<0$;

(II) $z(t)<0, z^{\prime}(t)>0,\left(a(t) z^{\prime}(t)\right)^{\prime}<0$,

for all $t \geq t_{1}$, where $t_{1} \geq t_{0}$ is sufficiently large.

Proof. Assume that $x(t)>0, x(\tau(t))>0$ and $x(\sigma(t))>0$ for all $t \geq t_{1}$ for some $t_{1} \geq t_{0}$. From the definition of $z(t)$ and equation (1.1), we have

$$
\left(a(t) z^{\prime}(t)\right)^{\prime}=-q(t) x^{\beta}(\sigma(t))<0 .
$$

Hence $a z^{\prime}$ is decreasing and of one sign for large $t$, that is, there exists a $t_{2} \geq t_{1}$ such that $z^{\prime}(t)>0$ or $z^{\prime}(t)<0$ for all $t \geq t_{2}$.

If $z^{\prime}(t)<0$ for $t \geq t_{2}$, then $a(t) z^{\prime}(t) \leq-d<0$ for $t \geq t_{2}$, where $d=-a\left(t_{2}\right) z^{\prime}\left(t_{2}\right)>0$. Thus, we have

$$
z(t) \leq z\left(t_{2}\right)-d \int_{t_{2}}^{t} \frac{\mathrm{d} s}{a(s)} .
$$

In view of condition 1.2 , we see that $\lim _{t \rightarrow \infty} z(t)=-\infty$. Now we consider the following two cases separately.

$\underline{\text { Case 1. }}$. If $x$ is unbounded, then there exists a sequence $\left\{t_{k}\right\}$ such that $\lim _{k \rightarrow \infty} t_{k}=\infty$ and $\lim _{k \rightarrow \infty} x\left(t_{k}\right)=\infty$ where $x\left(t_{k}\right)=\max \left\{x(s): t_{0} \leq s \leq t_{k}\right\}$. Since $\lim _{t \rightarrow \infty} \tau(t)=\infty, \tau\left(t_{k}\right)>t_{0}$ for all sufficiently large $k$ and $\tau(t) \leq t$, we have

$$
x\left(\tau\left(t_{k}\right)\right)=\max \left\{x(s): t_{0} \leq s \leq \tau\left(t_{k}\right)\right\} \leq \max \left\{x(s): t_{0} \leq s \leq t_{k}\right\}=x\left(t_{k}\right) .
$$

Hence

$$
z\left(t_{k}\right)=x\left(t_{k}\right)-p\left(t_{k}\right) x^{\alpha}\left(\tau\left(t_{k}\right)\right) \geq\left(1-p\left(t_{k}\right) x^{\alpha-1}\left(t_{k}\right)\right) x\left(t_{k}\right) \rightarrow \infty
$$


as $k \rightarrow \infty$ since $0<\alpha \leq 1$ and $p(t)$ is bounded, which contradicts the fact that $\lim _{t \rightarrow \infty} z(t)=-\infty$. $\underline{\text { Case 2. }}$. If $x$ is bounded then $z$ is also bounded, since $p(t)$ is bounded, which contradicts $\lim _{t \rightarrow \infty} z(t)=$ $-\infty$. Hence $z$ satisfies one of the cases (I) and (II). This completes the proof.

Lemma 2.2. Assume condition (1.2) holds. Let $x$ be a positive solution of equation (1.1) such that case (I) of Lemma 2.1 holds. Then

$$
x(t)>z(t)>R(t) a(t) z^{\prime}(t)
$$

for all $t \geq t_{1}$ and $z(t) / R(t)$ is eventually decreasing.

Proof. From the definition of $z$ and $\left(\mathrm{C}_{2}\right)$ we have $x(t)>z(t)$ for all $t \geq t_{1} \geq t_{0}$. In view of case (I), we obtain

$$
\begin{aligned}
z(t) & =z\left(t_{1}\right)+\int_{t_{1}}^{t} \frac{a(s) z^{\prime}(s)}{a(s)} \mathrm{d} s \\
& >R(t) a(t) z^{\prime}(t), \quad t \geq t_{1} .
\end{aligned}
$$

Furthermore,

$$
\left(\frac{z(t)}{R(t)}\right)^{\prime}=\frac{a(t) R(t) z^{\prime}(t)-z(t)}{a(t) R^{2}(t)}, \quad t \geq t_{1}
$$

By 2.3 , one obtains $\left(\frac{z(t)}{R(t)}\right)^{\prime}<0$ for all $t \geq t_{1}$. Thus, $\frac{z(t)}{R(t)}$ is strictly decreasing for all $t \geq t_{1}$. This completes the proof.

ThEOREM 2.1. Let $\beta<\alpha, \sigma(t)<\tau(t)$, and condition 1.2 hold. If

$$
\int_{t_{1}}^{\infty} q(t) R^{\beta}(\sigma(t)) \mathrm{d} t=\infty
$$

and

$$
\limsup _{t \rightarrow \infty} \int_{\tau^{-1}(\sigma(t))}^{t} \frac{1}{a(s)} \int_{s}^{t} \frac{q(u)}{p^{\frac{\beta}{\alpha}}\left(\tau^{-1}(\sigma(u))\right)} \mathrm{d} u \mathrm{~d} s>0
$$

then every solution of equation 1.1] is oscillatory.

P r o o f. Assume that there is a nonoscillatory solution $x$ of equation 1.1 , say $x(t)>0, x(\tau(t))>0$ and $x(\sigma(t))>0$ for all $t \geq t_{1} \geq t_{0}$. It follows from Lemma 2.1 that the corresponding function $z$ satisfies either (I) or (II).

Let $z(t)$ satisfy case (I). From the definition of $z$, we have

$$
x(t) \geq z(t)
$$

and

$$
x^{\beta}(\sigma(t)) \geq z^{\beta}(\sigma(t)) .
$$

Substituting above inequality in equation (1.1), we obtain

$$
\left(a(t) z^{\prime}(t)\right)^{\prime}+q(t) z^{\beta}(\sigma(t)) \leq 0 .
$$

Now using 2.2 in 2.6 and letting $w(t)=a(t) z^{\prime}(t)$, we see that $w(t)$ is a positive solution of the inequality

$$
w^{\prime}(t)+q(t) R^{\beta}(\sigma(t)) w^{\beta}(\sigma(t)) \leq 0, \quad t \geq t_{1} .
$$




\section{BLANKA BACULÍKOVÁ - B. SUDHA - K. THANGAVELU - E. THANDAPANI}

On the other hand, by 6. Theorem 3.9.3] condition (2.4) guarantees that (2.7) has no eventually positive solution, a contradiction.

Let $z(t)$ satisfy case (II) of Lemma 2.1. From the definition of $z$, we have

$$
x(\tau(t))>\left(-\frac{z(t)}{p(t)}\right)^{1 / \alpha} .
$$

Using (2.8) in equation (1.1), we obtain

$$
\left(a(t) z^{\prime}(t)\right)^{\prime}-\frac{1}{p^{\beta / \alpha}\left(\tau^{-1}(\sigma(t))\right)} q(t) z^{\beta / \alpha}\left(\tau^{-1}(\sigma(t))\right) \leq 0 .
$$

Since $z(t)$ is negative and increasing, we obtain $\lim _{t \rightarrow \infty} z(t)=d \leq 0$. We show that $d=0$. If not, then $d<0$ and $z(t) \leq d$ and $z\left(\tau^{-1}(\sigma(t))\right) \leq d$ for $t$ large enough. Therefore

$$
z^{\beta / \alpha}\left(\tau^{-1}(\sigma(t))\right) \leq d^{\beta / \alpha} .
$$

Integrating inequality $(2.9)$ from $t$ to $\infty$ and using (2.10) we have

$$
-a(t) z^{\prime}(t) \leq \int_{t}^{\infty} \frac{q(u)}{p^{\frac{\beta}{\alpha}}\left(\tau^{-1}(\sigma(u))\right)} z^{\beta / \alpha}\left(\tau^{-1}(\sigma(u))\right) \mathrm{d} u \leq d^{\beta / \alpha} \int_{t}^{\infty} \frac{q(u)}{p^{\frac{\beta}{\alpha}}\left(\tau^{-1}(\sigma(u))\right)} \mathrm{d} u .
$$

Integrating once more the last inequality from $t_{1}$ to $\infty$, we obtain

$$
z\left(t_{1}\right) \leq d^{\beta / \alpha} \int_{t_{1}}^{\infty} \frac{1}{a(u)} \int_{u}^{\infty} \frac{q(s)}{p^{\frac{\beta}{\alpha}}\left(\tau^{-1}(\sigma(s))\right)} \mathrm{d} s \mathrm{~d} u,
$$

which is a contradiction with 2.5 , because from 2.5 , we claim

$$
\limsup _{t \rightarrow \infty} \int_{t_{1}}^{\infty} \frac{1}{a(u)} \int_{u}^{\infty} \frac{q(s)}{p^{\frac{\beta}{\alpha}}\left(\tau^{-1}(\sigma(s))\right)} \mathrm{d} s \mathrm{~d} u=\infty .
$$

So, we have $\lim _{t \rightarrow \infty} z(t)=0$ and $z(t)$ is negative and increasing. Integrating 2.9 from $s$ to $t$ for $t>s$, we get

$$
-a(s) z^{\prime}(s) \leq \int_{s}^{t} \frac{q(u)}{p^{\frac{\beta}{\alpha}}\left(\tau^{-1}(\sigma(u))\right)} z^{\beta / \alpha}\left(\tau^{-1}(\sigma(u))\right) \mathrm{d} u .
$$

Integrating above inequality from $\tau^{-1}(\sigma(t))$ to $t$ for $s$ and using that $z(t)$ is increasing, we have

$$
z\left(\tau^{-1}(\sigma(t))\right)-z(t) \leq z^{\beta / \alpha}\left(\tau^{-1}(\sigma(t))\right) \int_{\tau^{-1}(\sigma(t))}^{t} \frac{1}{a(s)} \int_{s}^{t} \frac{q(u)}{p^{\frac{\beta}{\alpha}}\left(\tau^{-1}(\sigma(u))\right)} \mathrm{d} u \mathrm{~d} s
$$

or

$$
\frac{z\left(\tau^{-1}(\sigma(t))\right)}{z^{\beta / \alpha}\left(\tau^{-1}(\sigma(t))\right)} \geq \int_{\tau^{-1}(\sigma(t))}^{t} \frac{1}{a(s)} \int_{s}^{t} \frac{q(u)}{p^{\frac{\beta}{\alpha}}\left(\tau^{-1}(\sigma(u))\right)} \mathrm{d} u \mathrm{~d} s .
$$

Since $\frac{z\left(\tau^{-1}(\sigma(t))\right)}{z^{\beta / \alpha}\left(\tau^{-1}(\sigma(t))\right)}=\left|z\left(\tau^{-1}(\sigma(t))\right)\right|^{1-\beta / \alpha}$ and $1-\beta / \alpha>0$, we have

$$
\limsup _{t \rightarrow \infty} \int_{\tau^{-1}(\sigma(t))}^{t} \frac{1}{a(s)} \int_{s}^{t} \frac{q(u)}{p^{\frac{\beta}{\alpha}}\left(\tau^{-1}(\sigma(u))\right)} \mathrm{d} u \mathrm{~d} s \leq 0,
$$

which contradicts (2.5). This completes the proof of the theorem. 
THEOREM 2.2. Let $\beta=1$, and condition 1.2 hold. If

$$
\liminf _{t \rightarrow \infty} \int_{\sigma(t)}^{t} q(s) R(\sigma(s)) \mathrm{d} s>\frac{1}{\mathrm{e}},
$$

then every solution of equation (1.1) is either oscillatory or tends to zero as $t \rightarrow \infty$.

Proof. Assume that there exists a nonoscillatory solution $x$ of equation (1.1), say, $x(t)>0$, $x(\tau(t))>0$, and $x(\sigma(t))>0$ for all $t \geq t_{1} \geq t_{0}$ so that for $z$ one of the cases (I) and (II) holds.

Let $z(t)$ satisfy case (I) of Lemma 2.1. From the proof of case (I) of Theorem 2.1. we have for $\beta=1$ that $w(t)=a(t) z^{\prime}(t)$ is a positive solution of inequality

$$
w^{\prime}(t)+q(t) R(\sigma(t)) w(\sigma(t)) \leq 0 .
$$

On the other hand, by [6. Theorem 2.1.1] condition 2.12 guarantees that inequality 2.13 has no eventually positive solution, a contradiction.

Let $z(t)$ satisfy case (II) of Lemma 2.1. By $z<0$ and $z^{\prime}>0$, we see that

$$
\lim _{t \rightarrow \infty} z(t)=d \leq 0
$$

where $d$ is a finite constant. That is, $z$ is bounded and as in the proof of case (1) of Lemma 2.1. $x$ is also bounded. Therefore $\lim _{t \rightarrow \infty} x(t)=\ell, 0 \leq \ell<\infty$. We claim that $\ell=0$. If $\ell>0$, there is a sequence $\left\{t_{k}\right\}$ such that $\lim _{k \rightarrow \infty} t_{k}=\infty$ and $\lim _{k \rightarrow \infty} x\left(t_{k}\right)=\ell$. Now

$$
z\left(t_{k}\right)=x\left(t_{k}\right)-p\left(t_{k}\right) x^{\alpha}\left(\tau\left(t_{k}\right)\right)
$$

and so

Letting $k \rightarrow \infty$, we obtain

$$
x\left(\tau\left(t_{k}\right)\right)=\frac{1}{p^{\frac{1}{\alpha}}\left(t_{k}\right)}\left(x\left(t_{k}\right)-z\left(t_{k}\right)\right)^{\frac{1}{\alpha}} .
$$

$$
\ell \geq \lim _{k \rightarrow \infty} x\left(\tau\left(t_{k}\right)\right) \geq\left(\frac{\ell}{p}\right)^{\frac{1}{\alpha}} .
$$

Since $p \in(0,1)$, we see that $\ell=0$, that is, $\lim _{t \rightarrow \infty} x(t)=0$. This completes the proof of the theorem.

THEOREM 2.3. Let $\beta>1$, and condition (1.2) hold. If there exists a positive nondecreasing function $\rho(t)$ such that

$$
\lim _{t \rightarrow \infty} \sup \int_{t_{2}}^{t}\left[\rho(s) q(s)-\frac{a(\sigma(s))\left(\rho^{\prime}(s)\right)^{2}}{4 \beta K^{\beta-1} \rho(s) \sigma^{\prime}(s)}\right] \mathrm{d} s=\infty
$$

for any constant $K>0$, then every solution of equation (1.1) is either oscillatory or tends to zero as $t \rightarrow \infty$.

Pro of. Proceeding as in the proof of Theorem 2.1, we see that one of the cases of Lemma 2.1 holds for all $t \geq t_{1}$.

Case (I) Proceeding as in the proof of Theorem 2.1 (Case (I)), we obtain

$$
\left(a(t) z^{\prime}(t)\right)^{\prime}+q(t) z^{\beta}(\sigma(t)) \leq 0
$$

for all $t \geq t_{1}$. Define

$$
w(t)=\rho(t) \frac{a(t) z^{\prime}(t)}{z^{\beta}(\sigma(t))}, \quad t \geq t_{1}
$$


Then $w(t)>0$, and from 2.15, we have

$$
w^{\prime}(t) \leq-\rho(t) q(t)+\frac{\rho^{\prime}(t)}{\rho(t)} w(t)-\frac{\beta a(t) z^{\prime}(t)}{z^{\beta+1}(\sigma(t))} z^{\prime}(\sigma(t)) \sigma^{\prime}(t) \rho(t) .
$$

Since $a(t) z^{\prime}(t)$ is nonincreasing we have $a(\sigma(t)) z^{\prime}(\sigma(t)) \geq a(t) z^{\prime}(t)$ and so

$$
w^{\prime}(t) \leq-\rho(t) q(t)+\frac{\rho^{\prime}(t)}{\rho(t)} w(t)-\frac{\beta a(\sigma(t))\left(z^{\prime}(\sigma(t))\right)^{2}}{z^{\beta+1}(\sigma(t))} \sigma^{\prime}(t) \rho(t) .
$$

Using that $z(t)$ is positive increasing and $\beta>1$ there is a constant $K>0$ such that $z^{\beta-1}(t) \geq$ $K^{\beta-1}>0$ for all $t \geq t_{2} \geq t_{1}$ and the last inequality implies

$$
w^{\prime}(t) \leq-\rho(t) q(t)+\frac{\rho^{\prime}(t)}{\rho(t)} w(t)-\frac{\beta K^{\beta-1} \sigma^{\prime}(t)}{\rho(t) a(\sigma(t))} w^{2}(t) .
$$

Now using the completing the square, we have

$$
w^{\prime}(t) \leq-\rho(t) q(t)+\frac{a(\sigma(t))\left(\rho^{\prime}(t)\right)^{2}}{4 \beta K^{\beta-1} \rho(t) \sigma^{\prime}(t)}, \quad t \geq t_{2} .
$$

Integrating the last inequality from $t_{2}$ to $t$, we obtain

$$
\int_{t_{2}}^{t}\left[\rho(s) q(s)-\frac{a(\sigma(s))\left(\rho^{\prime}(s)\right)^{2}}{4 \beta K^{\beta-1} \rho(s) \sigma^{\prime}(s)}\right] \mathrm{d} s<w\left(t_{2}\right)<\infty .
$$

Take limsup as $t \rightarrow \infty$ in the last inequality, we obtain a contradiction to (2.14).

Case (II) In this case $z(t)<0$ and $z^{\prime}(t)>0$ for all $t \geq t_{1}$. Then proceeding as in case (II) of Theorem 2.2 we obtain $\lim _{t \rightarrow \infty} x(t)=0$. This completes the proof of the theorem.

\section{Examples}

In this section, we present three examples to illustrate the main results.

Example 1. Consider a second order neutral differential equation

$$
\left(x(t)-p x^{1 / 3}(t / 2)\right)^{\prime \prime}+8 t x^{1 / 5}(t / 3)=0, \quad t \geq 1,
$$

where $p \in(0,1)$ is a constant. Here $a(t)=1, p(t)=p, q(t)=8 t, \tau(t)=t / 2, \sigma(t)=t / 3$ for $t \geq t_{1}=1, \alpha=1 / 3, \beta=1 / 5$ and $R(t)=t-1$. Simple calculation shows that conditions 2.4 and (2.5) are satisfied. Therefore by Theorem 2.1 every solution of equation (3.1) is oscillatory.

Example 2. Consider a second order neutral differential equation

$$
\left(t\left(x(t)-p x^{1 / 3}(t / 2)\right)^{\prime}\right)^{\prime}+t x(t / 3)=0, \quad t \geq 1,
$$

where $p \in(0,1)$ is a constant. Here $a(t)=t, p(t)=p, q(t)=t, \tau(t)=t / 2, \sigma(t)=t / 3$ for $t \geq t_{1}=1, \alpha=1 / 3, \beta=1$ and $R(t)=\ln t$. Now one can easily verify that all conditions of Theorem 2.2 are satisfied. Hence every solution of equation 3.2 is either oscillatory or tends to zero as $t \rightarrow \infty$. 


\section{OSCILLATION OF SECOND ORDER EQUATION}

Example 3. Consider a second order neutral differential equation

$$
\left(t\left(x(t)-p x^{1 / 3}(t-\pi / 2)\right)^{\prime}\right)^{\prime}+\frac{1}{t} x^{3}(t-\pi)=0, \quad t \geq 1,
$$

where $p \in(0,1)$ is a constant. Here $a(t)=t, p(t)=p, q(t)=\frac{1}{t}, \tau(t)=t-\pi / 2, \sigma(t)=t-\pi$ or $t \geq t_{1}=1, \alpha=1 / 3, \beta=3$ and $R(t)=\ln t$. By taking $\rho(t)=1$, one can easily see that all conditions of Theorem 2.3 are satisfied, and hence every solution of equation (3.3) is either oscillatory or tends to zero as $t \rightarrow \infty$.

We conclude this paper with the following remark.

Remark 1. Note that Theorem 2.1 guarantees that every solution of equation 1.1 is oscillatory, and from other theorems we see that every solution is either oscillatory or tends to zero as $t \rightarrow \infty$. Therefore it is interesting to improve Theorems 2.2 , and 2.3 so that all solutions of equation (1.1) are oscillatory only. Further note that the results reported in $7,11,13,17$ cannot be applied to equations (3.1), 3.2) and (3.3) since the neutral term is not linear.

Acknowledgement. The paper is supported by the grant project KEGA 037TUKE-4/2020.

\section{REFERENCES}

[1] AGARWAL, R. P.-BOHNER, M.-LI, T.: Nonoscillation and Oscillation Theory of Functional Differential Equations, Marcel Dekker, New York, 2004.

[2] AGARWAL, R. P.-BOHNER, M.-LI T.-ZHANG, C.: Oscillation of second order differential equations with a sublinear neutral term, Carpathian J. Math. 30 (2014), 1-6.

[3] AGARWAL, R. P.-GRACE, S. R.: Oscillation theorems for certain neutral differential equations, Comput. Math. Appl. 38 (1999), 1-11.

[4] BACULÍKOVÁ, B.-DŽURINA, J.: Oscillation theorems for second order nonlinear neutral differential equations, Comput. Math. Appl. 61 (2011), 94-99.

[5] DONG, J. G.: Oscillation behavior of second order nonlinear neutral differential equations with deviating arguments, Comput. Math. Appl. 59 (2010), 3710-3717.

[6] ERBE, L. H.-KONG, Q.-ZHANG, B. G.: Oscillation Theory For Functional Differential Equations, Marcel Dekker, New York, 1995.

[7] GRACE, S. R.: Oscillatory behavior of second-order nonlinear differential equations with a nonpositive neutral terms, Mediterr. J. Math. 14 (2017), Art. 229.

[8] GYORI, I.-LADAS, G.: Oscillation Theory of Delay Differential Equations with Applications, Clarendon Press, Oxford, 1991.

[9] HALE, J. K.: Theory of Functional Differential Equations, Springer-Verlag, New York, 1987.

[10] LI T.-AGARWAL R.P.-BOHNER M.: Some oscillation results for second order neutral differential equations, J. Indian Math. Soc. 79 (2012), 97-106.

[11] LI, Q.-WANG, W.-CHEN, F-LI, T.: Oscillation of second order nonlinear delay differential equations with nonpositive neutral coefficients, Adv. Difference Equ. 2015 (2015), Art. Id. 35.

[12] LIN, X.: Oscillation of second order nonlinear neutral differential equations, J. Math. Anal. Appl. 309 (2005), $442-452$.

[13] QIN, H.-SHANG, N.-LU, Y.: A note on oscillation criteria of second order nonlinear neutral delay differential equations, Comput. Math. Appl. 56 (2008), 2987-2992.

[14] TAMILVANAN, S.-THANDAPANI, E.-DŽURINA, J.: Oscillation of second order nonlinear differential eqation with sublinear neutral term, Diff. Equ. Appl. 9 (2017), 29-35.

[15] TAMILVANAN, S.-THANDAPANI, E.-GRACE, S. R.: Oscillation theorems for second order nonlinear differential equation with a nonlinear neutral term, Int. J. Dyn. Syst. Differ. Equ. 7 (2017), 316-327.

[16] THANDAPANI, E.-RAMA, R.: Comparison and oscillation theorems for second order nonlinear neutral differential equations, Serdica Math. J. 39 (2013), 1-16.

[17] WONG, J. S. W: Necessary and sufficient conditions for oscillation of second order neutral differential equations, J. Math. Anal. Appl. 252 (2000), 342-352. 


\section{BLANKA BACULÍKOVÁ - B. SUDHA - K. THANGAVELU - E. THANDAPANI}

Received 15. 12. 2020

Accepted 27. 2. 2021
* Department of Mathematics

Faculty of Electrical Engineering and Informatics

Technical University of Košice

SLOVAKIA

E-mail: blanka.baculikova@tuke.sk

** Department of Mathematics

Institute of Science and Technology

Kattankulathur

INDIA

E-mail: sudhabala10@gmail.com

*** Department of Mathematics

Pachiyappas College

Chennai

INDIA

E-mail: kthangavelu14@gmail.com

**** Ramanujan Institute For Advanced Study in Mathematics University of Madras

Chennai

INDIA

E-mail: ethandapani@yahoo.co.in 